\title{
Analysis of the optical design for the SAFIR telelscope
}

\section{Paul Goldsmith, Behrouz Khayatian, Matt Bradford, Mark Dragovan, Daniel Hoppe, et al.}

Paul Goldsmith, Behrouz Khayatian, Matt Bradford, Mark Dragovan, Daniel Hoppe, William Imbriale, Roger Lee, Chris Paine, Philip Turner, Harold Yorke, Jonas Zmuidzinas, "Analysis of the optical design for the SAFIR telelscope," Proc. SPIE 6265, Space Telescopes and Instrumentation I: Optical, Infrared, and Millimeter, 62654A (16 June 2006); doi: $10.1117 / 12.670051$

Event: SPIE Astronomical Telescopes + Instrumentation, 2006, Orlando, Florida, United States 


\title{
ANALYSIS OF THE OPTICAL DESIGN FOR THE SAFIR TELESCOPE
}

\author{
Paul Goldsmith, Behrouz Khayatian, Matt Bradford, Mark Dragovan, Daniel Hoppe, William \\ Imbriale, Roger Lee, Chris Paine, Philip Turner, and Harold Yorke \\ Jet Propulsion Laboratory, 4800 Oak Grove Drive, Pasadena CA 91109 \\ and \\ Jonas Zmuidzinas \\ California Institute of Technology MS 320-47, Pasadena CA 91125
}

\begin{abstract}
SAFIR, the Single Aperture Far Infra Red Observatory, is a very powerful space mission that will achieve background-limited sensitivity in the far infrared-submillimeter spectral region. Many processes of enormous interest to astronomers can best be studied in this wavelength range, but require the demanding combination of high sensitivity, good angular resolution, and spectroscopic capability. SAFIR is a $10 \mathrm{~m}$ class telescope offering good angular resolution, cooled to below $5 \mathrm{~K}$ in order to achieve background-limited sensitivity, and equipped with a complement of large-format cameras and broadband spectrometers. Successful operation of such a facility is critically dependent on achieving the level of sensitivity expected, but this is rendered difficult by potential pickup from unwanted sources of radiation. This problem is exacerbated by the fact that the emission from the optical system itself is minimal due to its low temperature, thus emphasizing the importance of minimizing pickup from unwanted astronomical sources of radiation, including the emission from dust in our solar system (analogous to the zodiacal light, hence "zodi"), and the emission from warm dust in the Milky Way (Galactic "cirrus").
\end{abstract}

The extreme sensitivity of SAFIR to these unwanted sources of radiation makes it essential to understand the relative sensitivity of the telescope/detector system to radiation coming from angles far outside the main beam, and to develop designs which minimize this pickup. In this paper we analyze in some detail the relative telescope sensitivity (referred to as the antenna pattern by microwave engineers) for different designs of SAFIR. These calculations include edge diffraction from the secondary and primary reflector, and also the effect of blockage by the secondary and blockage and scattering by support legs in a symmetric system. By convolving the antenna pattern with the brightness of the sky due to the zodi and cirrus, we can calculate the power received when the antenna is pointed in any specified direction. We can also compare the undesired pickup for different designs, in particular symmetric vs. asymmetric (off-axis or unblocked) antenna configurations. These considerations are vital for achieving the most efficient SAFIR design possible, in terms of achieving maximum sensitivity while being able to observe over a large fraction of the sky.

Space Telescopes and Instrumentation I: Optical, Infrared, and Millimeter, edited by John C. Mather, Howard A. MacEwen, Mattheus W.M. de Graauw, Proc. of SPIE Vol. 6265, 62654A, (2006) · 0277-786X/06/\$15 - doi: 10.1117/12.670051

Proc. of SPIE Vol. 6265 62654A-1 


\section{SAFIR SCIENTIFIC OBJECTIVES AND REQUIREMENTS}

SAFIR will operate in the $20 \mu \mathrm{m}$ to $800 \mu \mathrm{m}$ wavelength range (frequencies between $375 \mathrm{GHz}$ and $15 \mathrm{THz}$ ). This region includes a number of important spectral lines, and is the region that includes a large fraction of the thermal emission of dust in the interstellar medium. The scientific objectives of SAFIR are to exploit the diagnostic ability of the submillimeter-far infrared region that become available with dramatically enhanced sensitivity and good angular resolution. The detailed scientific case has been discussed at some length in other documents (Harvey et al. 2003; Benford et al. 2004; Lester et al. 2005), and will not be repeated here. In minimal form, SAFIR's key science objectives are to

- Probe the earliest epochs of metal enrichment and observe the galaxy forming universe before metals. Understand the origin of dust grains in the universe.

- Resolve and dissect the far infrared cosmic background - trace formation and evolution of the star forming active galaxies since the dawn of the universe, and measure the history of star formation.

- Explore the connection between embedded nuclear black holes and their host galaxies. Understand the relationship of active nuclei to galaxy formation.

- Track the chemistry of life. Follow prebiotic molecules, ices, and minerals from clouds to nascent solar systems.

- Identify young solar systems from debris disk structure and map the birth of planetary systems from deep within obscuring envelopes. Assess the degree of bombardment they face, and the degree of habitability.

The region of the electromagnetic spectrum in which SAFIR will have its major impact is accessible from the ground only from the highest sites with minimum water vapor above them. Even from these, there are only selected, relatively narrow frequency ranges in which the atmospheric transmission exceeds 0.5 . Even in these "windows" the appreciable atmospheric opacity means that the emission from the atmosphere is large. To achieve good sensitivity, then, requires first that one's telescope be above the earth's atmosphere. An airplane-based telescope improves the situation, but the integration time is limited, restricting, in particular, the ability to carry out large surveys. Highaltitude balloons can achieve even better atmospheric performance, but again only for modest flight durations. To carry out large-scale surveys of an appreciable fraction of the sky thus requires a spaceborne telescope.

The ultimate sensitivity of any telescope + detector system is set by the sky background itself. In this case, we mean the true astronomical background which one encounters when observing in a particular direction. The emission from any background includes fluctuations due to the quantized nature of the photons emitted. To make the background itself limit the sensitivity of the system, the average power emitted by any sources which can be eliminated (such as the earth's atmosphere discussed above, as well as the telescope) must be less than the average power received from the astronomical background. For SAFIR, the main contributions to the astronomical background are shown in Figure 1 (from Benford et al. 2004). They include 
- The cosmic background radiation -has a blackbody spectrum at a temperature of $2.7 \mathrm{~K}$ and is uniform in all directions. This source is dominant at wavelengths longer than $600 \mu \mathrm{m}$.

- The Galactic cirrus - has a grey body spectrum (blackbody multiplied by an emissivity which is less than unity) from dust grains in the interstellar medium of our Galaxy, characterized by temperature of $\sim 20 \mathrm{~K}$ and which is restricted largely to the plane of the Milky Way. In this direction, the cirrus is dominant at wavelengths between $150 \mu \mathrm{m}$ and $400 \mu \mathrm{m}$

- The zodiacal emission - radiation from dust grains in the solar system. This component is largely from the plane of the ecliptic. It is the dominant contributor at wavelengths less than $150 \mu \mathrm{m}$ if one is observing a source in the region of the sky which falls in plane of our solar system.

As illustrated in Figures 2 and 3, it is important to recall that these components have very different angular distributions on the sky, so their contributions will depend on the relative sensitivity of the telescope to radiation incident from different directions, as well as the direction in which the telescope is pointing.

In order to be limited by the astronomical "sky background", the power emitted by the telescope must be less than the sum of these various contributions. Only the cosmic background radiation is isotropic, while the others depend on the telescope behavior and pointing direction. Taking the viewpoint that all of these contribute simultaneously to the background, one derives the requirement that the telescope (primary and secondary, as well as any other optical elements) must be cooled to a temperature of $4 \mathrm{~K}$ or less, assuming the combined emissivity of all of the elements is 0.05 . Due to the very rapid fall-off in blackbody emission at wavelengths shorter than the peak, the upper limit to the temperature cannot be relaxed significantly even if the emissivity is reduced to a minimal value.

In addition to cooling the optics, achieving maximum sensitivity demands that detector noise levels be below those set by the background(s) discussed above. This limit depends on the bandwidth involved, and so detectors for spectroscopy (with fractional resolution $\delta \mathrm{f} / \mathrm{f}<10^{-3}$ ) must have much better performance in order to achieve background-limited performance than do those for broadband photometry. Thus, the actual background level that will characterize SAFIR on orbit will affect requirements for detectors as well as the ultimate sensitivity that can be achieved.

\section{MODELING THE RADIATION PATTERN OF THE SAFIR TELESCOPE}

The SAFIR antenna and spacecraft are complex structures, and calculating the sensitivity of the telescope plus detector as a function of angle is a challenging problem, because the sources of interest cover (including the comic background radiation) the entire celestial sphere. At this point we have restricted ourselves to the electromagnetic problem of diffraction only from the telescope itself, which we define as including the primary reflector, secondary reflector, and support legs. The geometry of the symmetric system shown in Figure 4 derives from that developed in the NASA Vision Mission Study (VMS; Lester 2005). It includes a primary reflector $10 \mathrm{~m}$ in diameter with focal length of $15 \mathrm{~m}$, a secondary reflector $2.96 \mathrm{~m}$ in diameter, and a final back focal distance of $2.8 \mathrm{~m}$.

The relatively large size of the secondary reflector was a result of the relatively large field of view required for the system. The VMS study specified a primary made of 7 mosaiced hexagonal 
segments, but we have for our analysis treated this as a circular aperture. We have also ignored the small difference between the Ritchey-Chrétien surfaces and those of the standard Cassegrain configuration we have modeled.

An important requirement is that our calculations describe the radiation pattern over the full celestial sphere, since we want to determine the contributions from the various extended sources of radiation described above, as well as possibly that from the moon, sun, and Earth, which can be in arbitrary locations relative to the main beam. This makes it impractical to use physical optics (PO) for the entire calculation, as the surface would have to be divided into regions $\leq \lambda / 2$ in size, which would total approximately $4 \times 10^{8}$ in number for a wavelength of $1 \mathrm{~mm}$, scaling as $\lambda^{-2}$. We have thus combined a Geometrical Optics and Geometrical Theory of Diffraction (GO/GTD) calculation for large angles, with a physical optics calculation for angles within 1 degree of boresight. With this restriction, the gridding of the radiating surface can be much coarser, thus drastically reducing the computation time, and we also avoid problems with ill-behaved GO/GTD results at caustics. We used the GRASP9 software package from TICRA to carry out the calculations, running on a fast, dual-processor PC.

\section{RESULTS FOR THE SYMMETRIC CONFIGURATION}

Figure 5 illustrates some of the contributions to the total radiation pattern that are calculated using the ray optics + GTD approach. This does not include the calculation of scattering by the secondary reflector support struts, which was treated by illuminating the struts with a plane wave. This is accurate in the sense that the struts extend beyond the edge of the primary reflector and there is thus no spherical wave blockage, but it does not explicitly include the tapering of the electric field as a function of radius. For the PO portion of the calculation, we used an equivalent feed located at the prime focus to illuminate the primary reflector with a Gaussian pattern falling to $-12 \mathrm{~dB}$ at the reflector edge. We also include the shadowing by the secondary reflector and the three $20 \mathrm{~cm}$ diameter reflector support struts.

A feeling for the magnitudes of the different components in the radiation pattern can be gained from a simple Gaussian beam analysis of the equivalent aperture plane electric field distribution (Goldsmith 1998). The spillover efficiency is 0.94 , meaning that $6 \%$ of the energy of the beam is in a cone with inner angular limit of $3.9^{\circ}$, the angular radius of the secondary reflector. The taper efficiency for the completely unblocked aperture is 0.87 ; this is the loss in directivity due to the Gaussian illumination with $12 \mathrm{~dB}$ edge taper that we have used. The blockage efficiency due to the secondary reflector is 0.71 . This relatively low value is a direct reflection of the large secondary reflector diameter needed to satisfy imaging requirements. The geometric blockage of the secondary support struts is 0.027 , and the loss in on-axis gain that results is twice this, so that the strut blockage efficiency is 0.95 . Combining all of these contributions yields an aperture efficiency of 0.55 . The above values are in very good agreement with the detailed diffraction calculations. They do not include the effects of any surface roughness, imperfect alignment, or panel gaps.

The angular distributions of the various components can be considered individually in detail based on the diffraction calculations discussed above. To understand where the radiation is actually going we present a selection of the calculated contributions to the radiation pattern in Figures $6-9$. These 
calculations have been carried out at a wavelength of $1 \mathrm{~mm}$. This is the long-wavelength limit for SAFIR, and is small compared to all relevant dimensions. Going to shorter wavelengths would significantly increase the computation time, but will be carried out for selected cases.

\section{FUTURE WORK}

Having the radiation pattern over the full $4 \pi$ sr of the sky, we can compute the coupling to the zodiacal and Galactic emission at different wavelengths, as shown in Figure 2 and 3. This will be a sensitive function of the boresight pointing direction, and will define the background as a function of source direction. The analysis of the symmetric design for SAFIR indicates that the secondary blockage, and more particularly the strut scattering, puts a significant fraction of the energy in the telescope beam at large angles from the pointing direction. This would have the effect of constraining the range of directions which can be observed while holding the background radiation pickup to an acceptable level. A design with lower large-angle from boresight response is clearly desirable.

In Figure 10 we show a conceptual off-axis design for the SAFIR system. This is based on a faster primary focal ratio, with $\mathrm{f} / \mathrm{D}=0.68$. The primary and secondary reflectors are perturbed from the conic sections of a conventional Gregorian system into biconic forms, having different radii of curvature in the plane of the offset and in the plane perpendicular to this. This design easily satisfies the requirements on imaging field of view, which are $100 \times 100$ diffraction limited pixels. There will be spillover and diffraction at the edge of the secondary reflector, which now is in a quite different direction from that of the main beam. There is also diffraction at the edge of the primary reflector, but there is no shadowing from the secondary or from support struts. We thus anticipate a considerably higher efficiency, and lower total fractional power at large angles from boresight, which will significantly reduce the power picked up from the Galactic and zodiacal dust emission. A detailed analysis of this improved design is currently underway.

This research described in this paper has been carried out at the Jet Propulsion Laboratory, California Institute of Technology, under a contract with the National Aeronautics and Space Administration. We thank André Girerd for his input to the project and suggestions that improved this paper. 


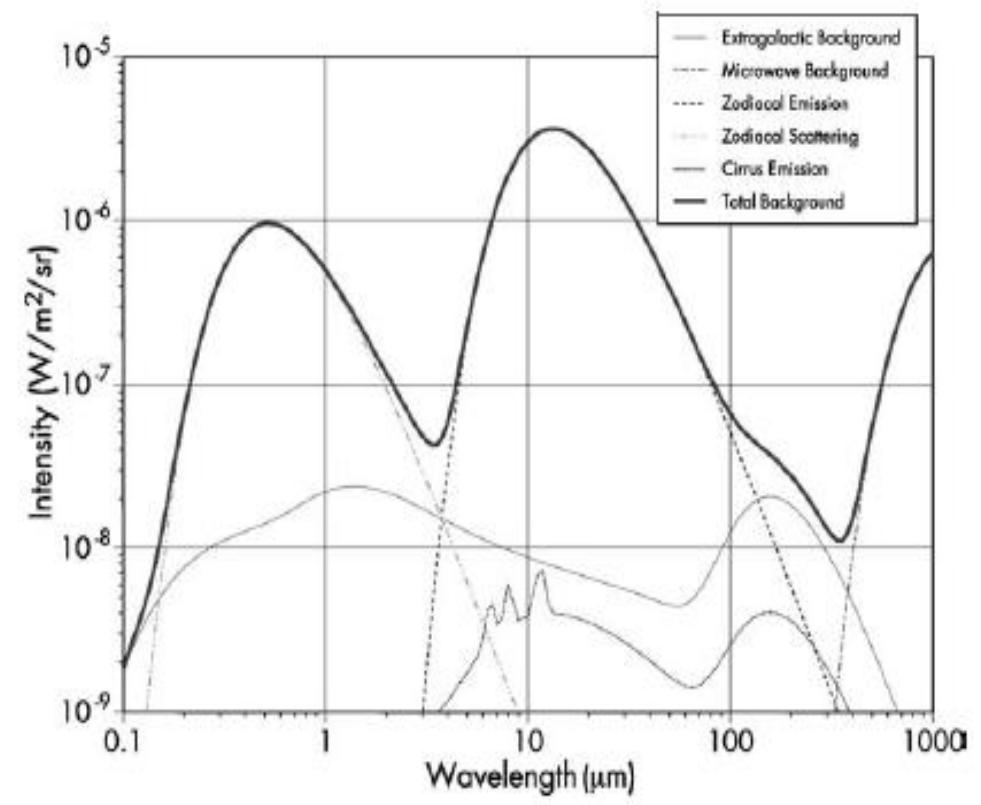

Figure 1 - Contributions to the background emission that will affect SAFIR sensitivity. The incident intensity is plotted as a function of wavelength. The region between $\sim 80 \mu \mathrm{m}$ and $500 \mu \mathrm{m}$ is characterized by particular low background emission - making the sky appear relatively "dark" in this wavelength range. From Benford et al. (2004). 


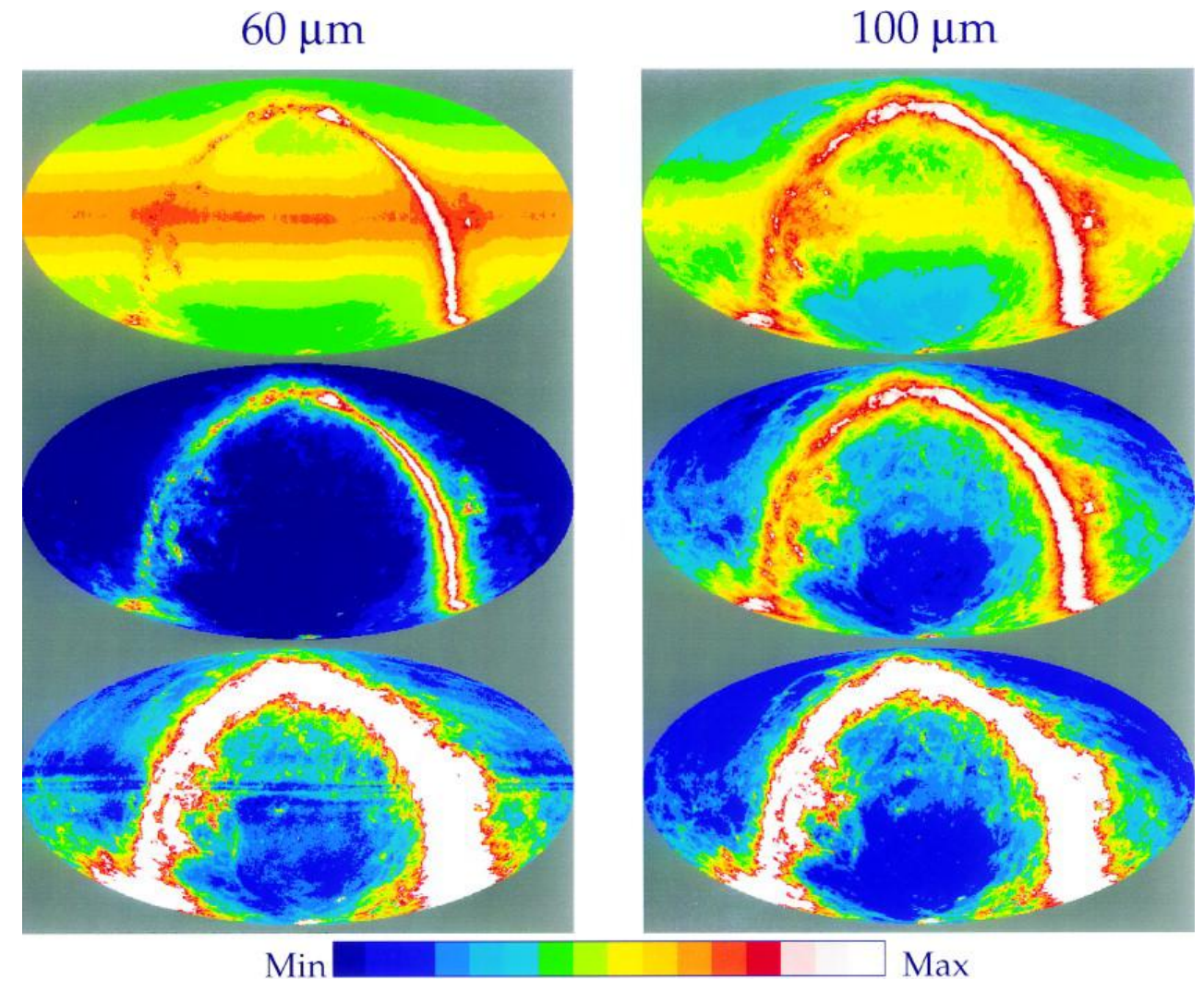

Figure 2. Maps of different components of the sky background measured at $60 \mu \mathrm{m}$ (left) and 100 $\mu \mathrm{m}$ (right) wavelengths by the DIRBE instrument on the COBE satellite (Kelsall et al. 1998). In each column the top image shows the incident signal as function of angle, on a logarithmic scale. The coordinate system is such that the plane of the ecliptic runs horizontally through the middle of the figure. The zodiacal emission is prominently visible as the horizontally stratified color coding of the intensity peaking in the ecliptic plane and varying in the perpendicular direction. The middle image in each column is the signal from the sky after removing a best-fit model of the zodiacal emission, again plotted on a logarithmic scale. The remaining dominant feature is the Galactic plane. The lower image in each column is the same as the middle image, but with a linear scale that emphasizes defects in model subtraction. The cosmic microwave background signal is very weak and uniform, so is not readily visible in these images, or in those of Figure 3. The Galactic dust emission has a very different distribution on the sky than does the zodiacal emission, which adds to the problem of calculating the total background picked up when SAFIR is pointing in a particular direction on the sky. 


\section{$140 \mu \mathrm{m}$}

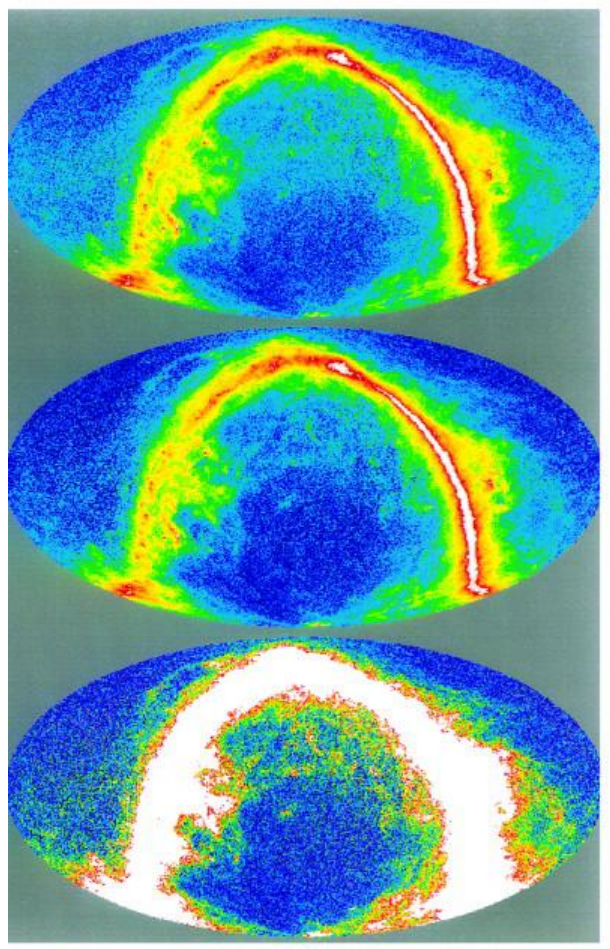

Min

\section{$240 \mu \mathrm{m}$}

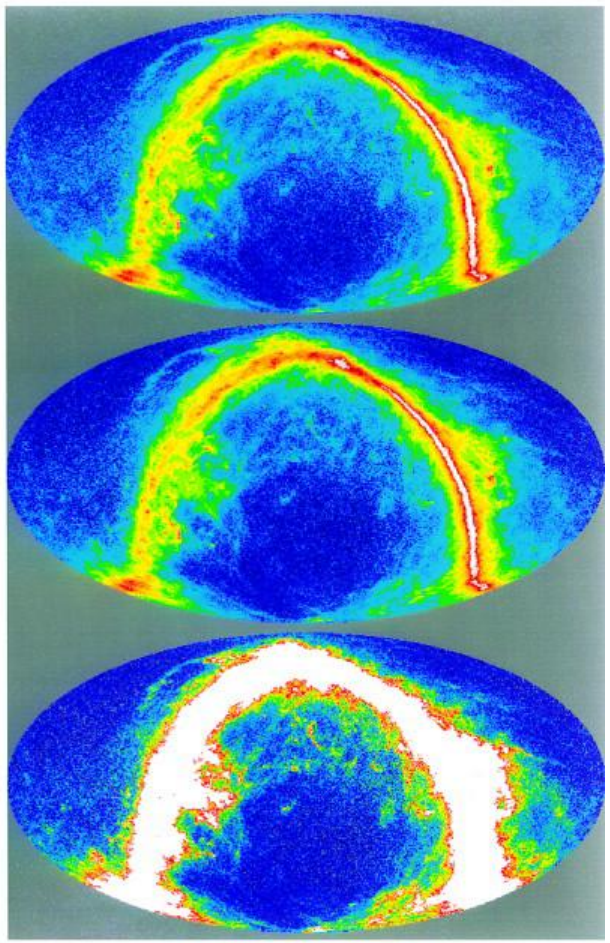

Max

Figure 3. As Figure 2, but wavelengths of $140 \mu \mathrm{m}$ (left) and $240 \mu \mathrm{m}$ (right). At these relatively long wavelengths, the zodiacal emission is no longer visible, leaving the Galactic dust as the dominant source. From Kelsall et al. (1998). 


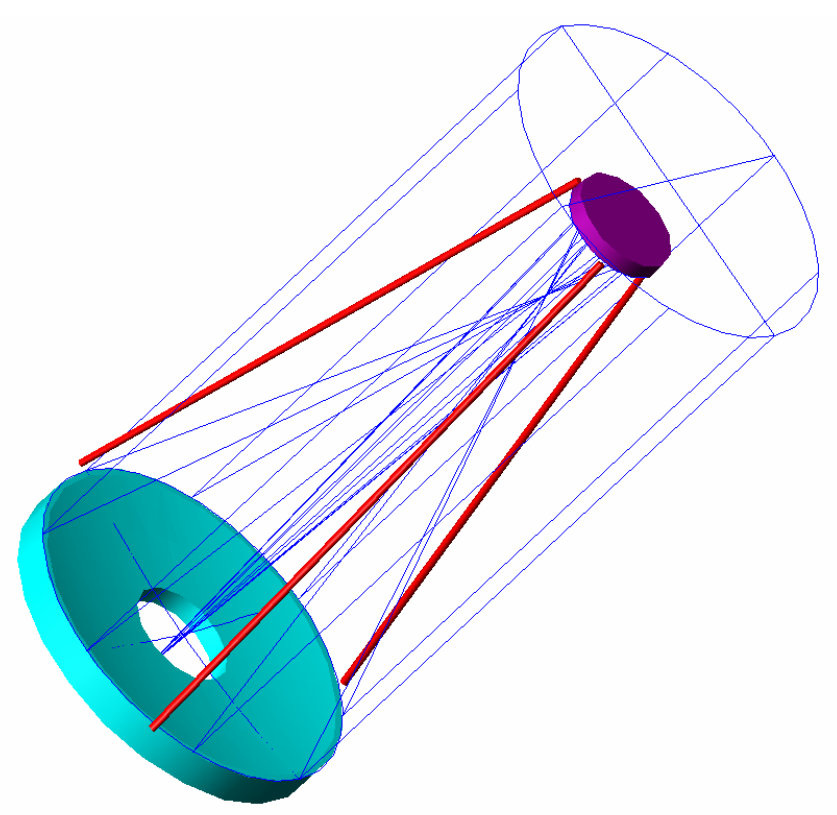

Figure 4. Schematic of on-axis SAFIR telescope configuration analyzed in the present study. The primary reflector diameter is $10 \mathrm{~m}$ and the secondary reflector diameter is $3 \mathrm{~m}$.

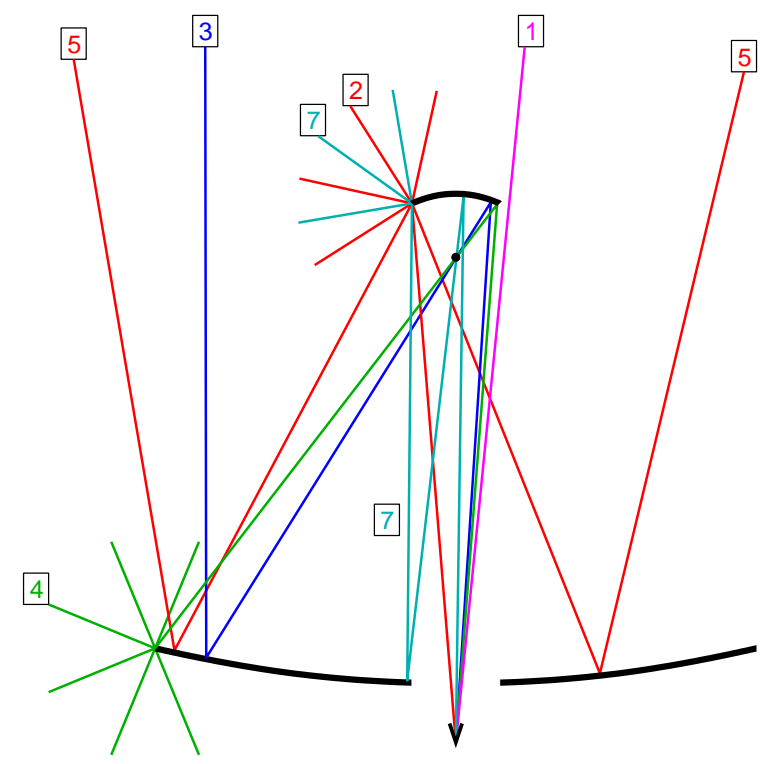

Figure 5. Contributions to the total radiation pattern of the SAFIR telescope using ray techniques by combining ray techniques with the Geometrical Theory of Diffraction (GTD). The most important are (1) Spillover past the secondary; (2) Diffraction from edge of the secondary; (3) Reflection from secondary and then from primary - the main beam; (4) Reflection from secondary and diffraction from the primary edge; (5) Diffraction from edge of the secondary, then reflection from the primary; (6) Diffraction from edge of the secondary and primary (not shown); (7) Reflection from the secondary, reflection from the primary, and diffraction from edge of the secondary. 


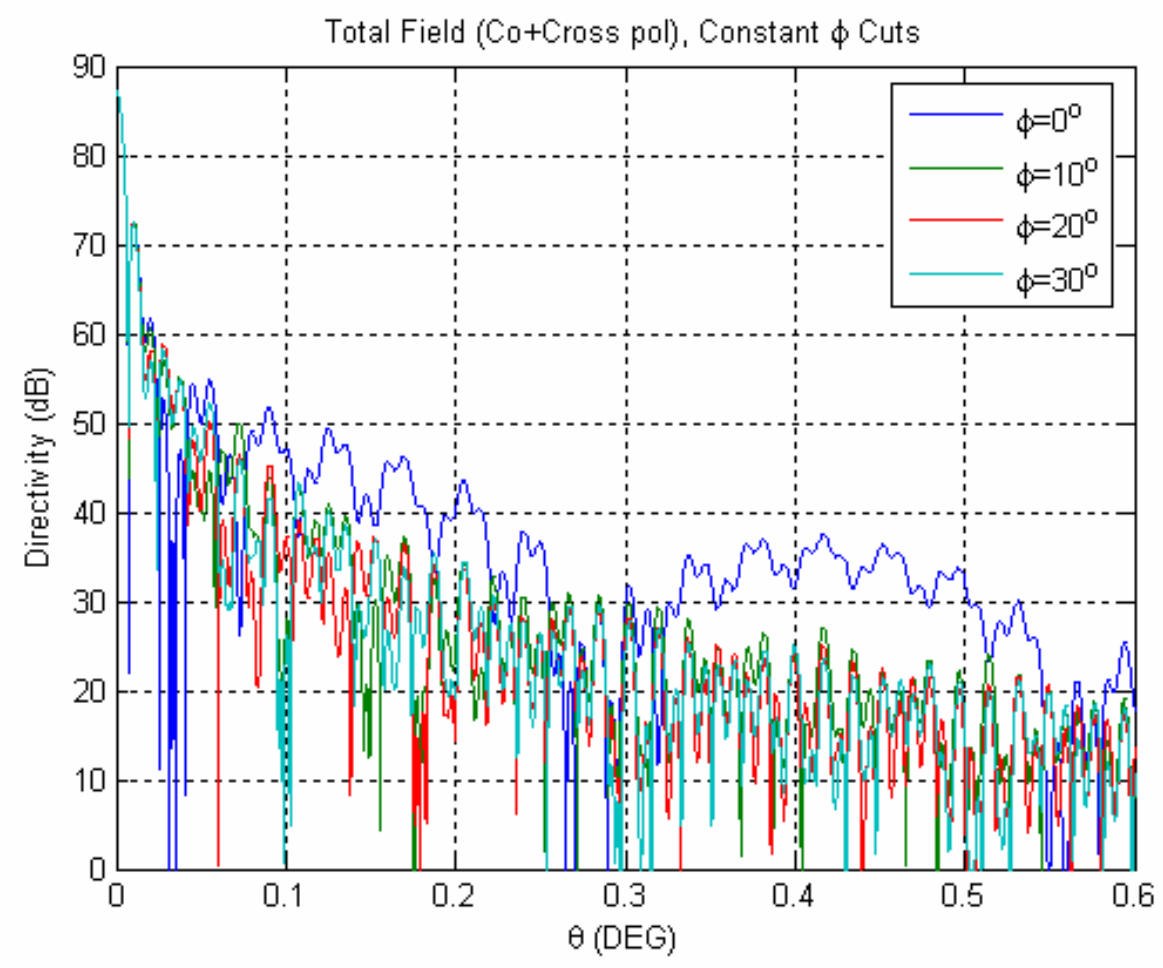

Figure 6. Cuts through power pattern of SAFIR antenna as function of off-boresight angle, made at four different azimuth angles at wavelength of $1 \mathrm{~mm}$ with no surface errors. The main beam peak directivity is $87.2 \mathrm{~dB}$, corresponding to an aperture efficiency of 0.55 . The main lobe and first main reflector diffraction sidelobe are clearly visible at all azimuth angles. The diffraction beam from the secondary reflector has a characteristic width of $0.02^{\circ}$, which is present at all azimuths, but which is most clearly visible at $\varphi=30^{\circ}$, where it interferes with the diffraction from the secondary reflector support struts. The $20 \mathrm{~cm}$ width of the strut produces the characteristic $0.3^{\circ}$ wide variations as a function of $\theta$. 


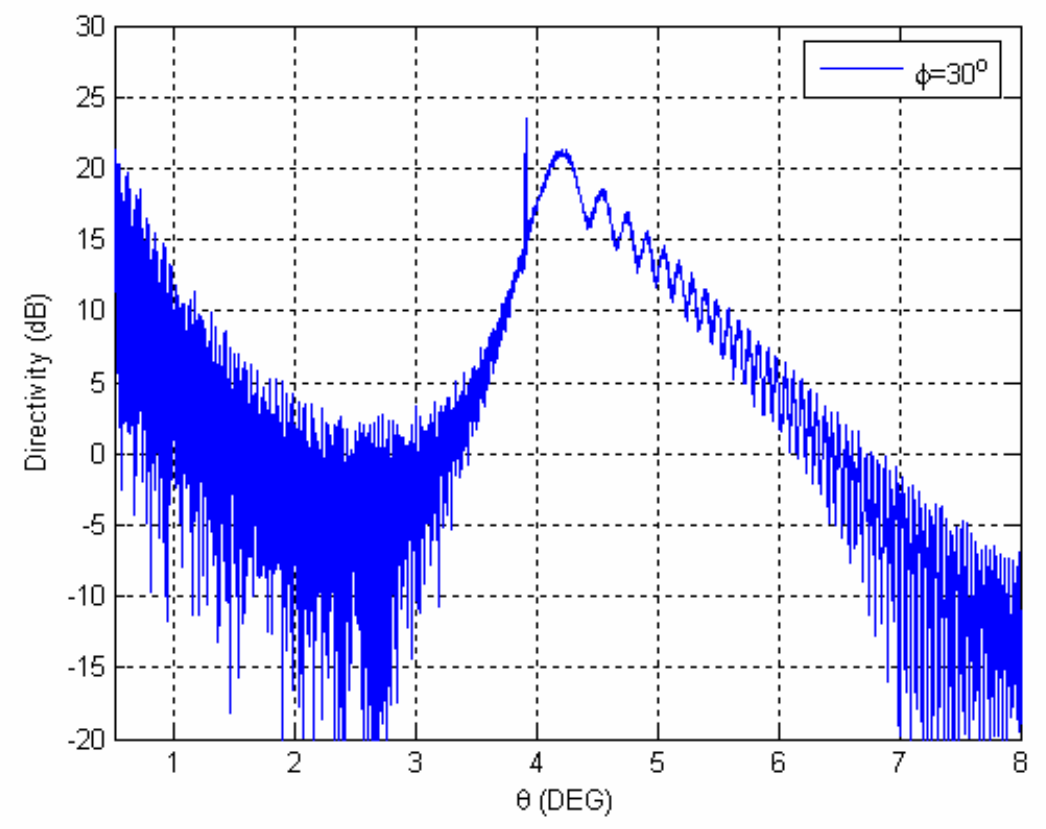

Figure 7. Radiation pattern at azimuth angle of $30^{\circ}$ showing the spillover (contribution [1] in Figure 5) and secondary reflector edge diffraction (contribution [2]). The edge of the secondary is at an angle of $3.9^{\circ}$. In the absence of diffraction, the radiation pattern at larger angles would be only the Gaussian beam pattern of the feed.

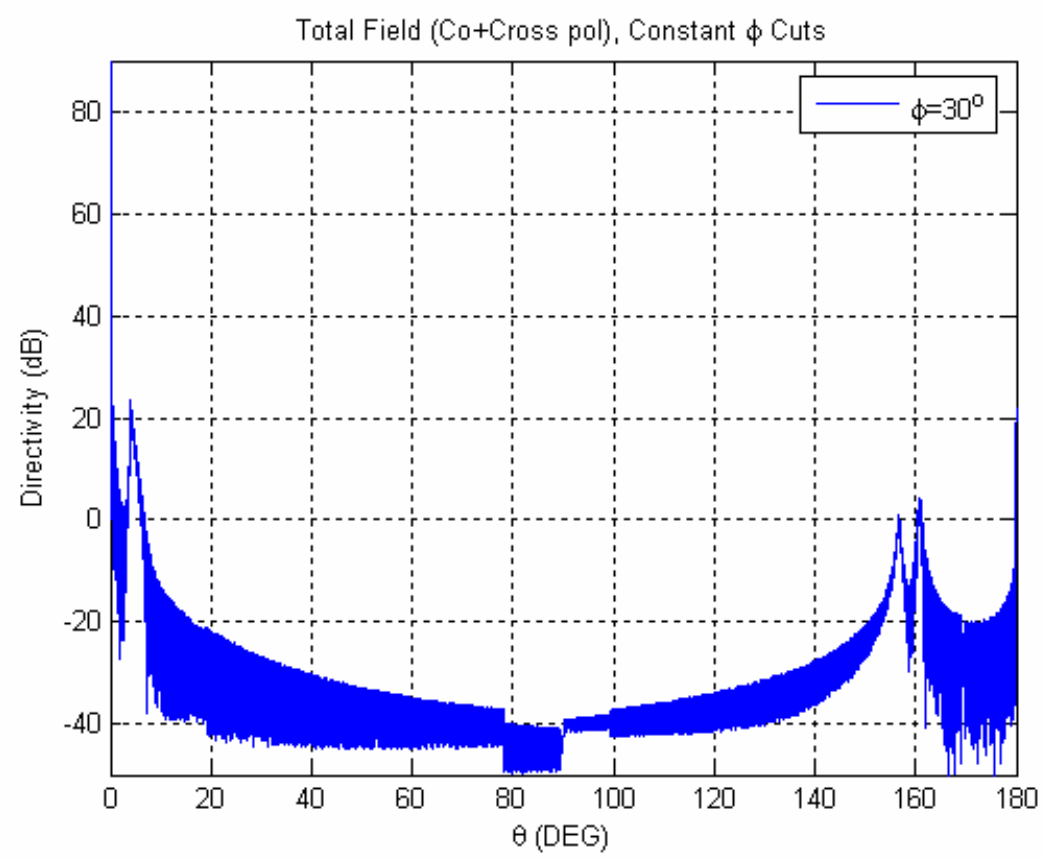

Figure 8. Large angle radiation pattern of SAFIR antenna. The peak near $4^{\circ}$ is due to spillover and secondary reflector edge diffraction as shown in Figure 7 . The peaks near $160^{\circ}$ are large-angle scattering from edges of primary and secondary reflectors (contributions [2] and [7]). Note the very low level of much of this pattern, relative to the boresight peak of $\sim 87 \mathrm{~dB}$. 


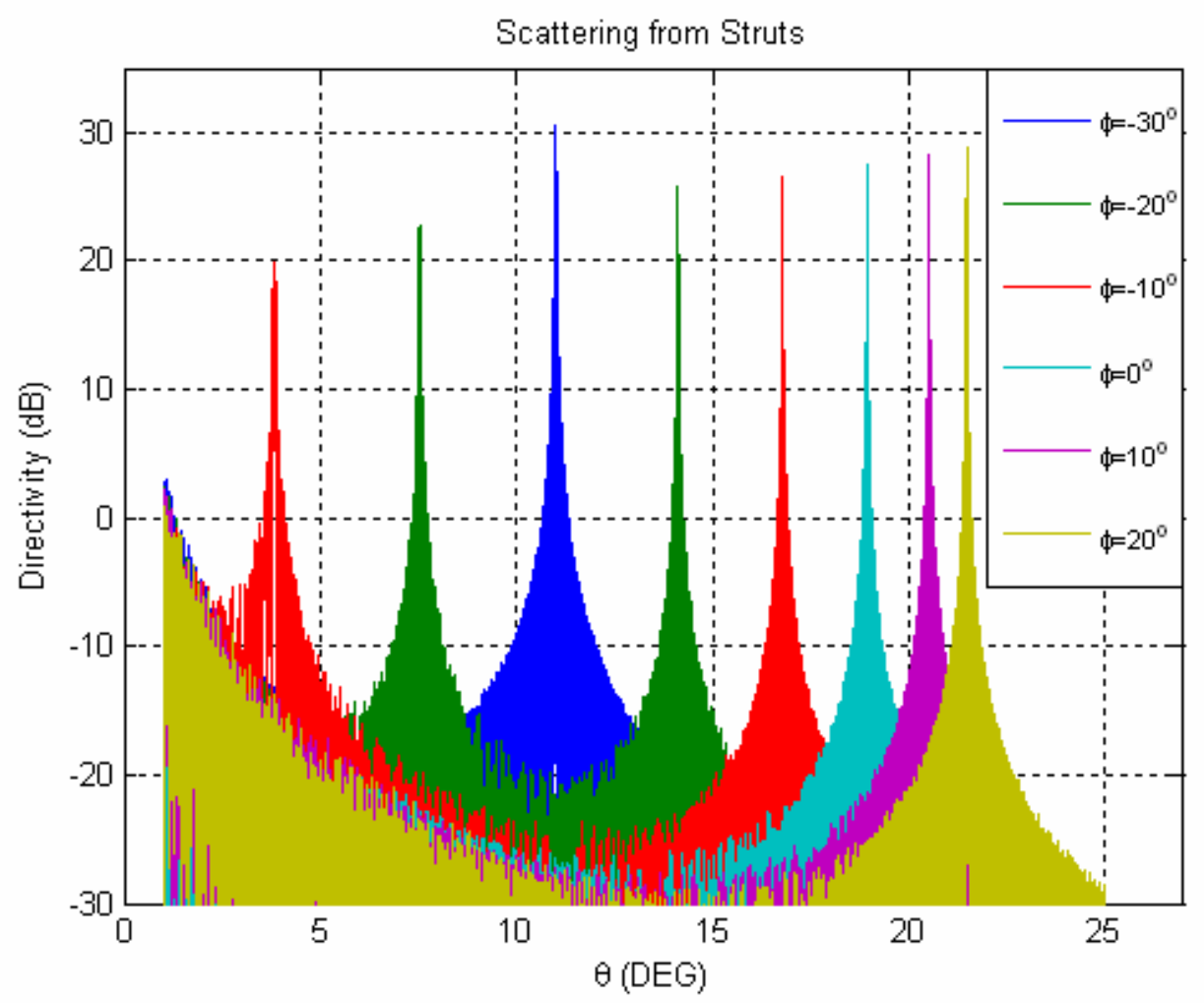

Figure 9. Contributions to the radiation pattern due to scattering from the secondary reflector support struts. This is the radiation scattered from the plane wave radiated by the primary reflector by the three support struts, which are modeled as perfectly conducting cylinders $20 \mathrm{~cm}$ in diameter. The support struts run from the edge of the secondary reflector to the edge of the primary reflector, and are inclined by $\sim 11^{\circ}$ relative to the axis of symmetry of the system. The scattering is distributed over all values of $\varphi$, but is restricted to $\theta<22^{\circ}$. 


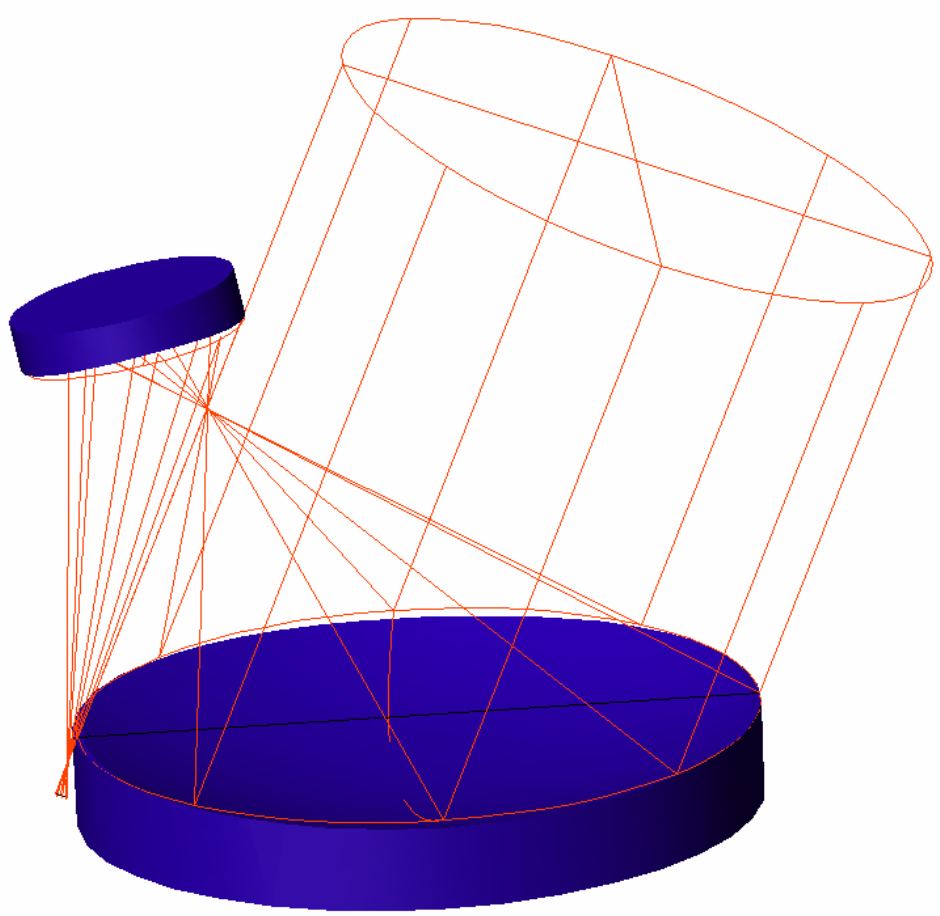

Figure 10. Schematic of off-axis, unblocked geometry for SAFIR antenna. The primary reflector diameter is again $10 \mathrm{~m}$, the secondary reflector diameter is $3 \mathrm{~m}$, and the off-axis angle is 45 degrees. The reflector surfaces are perturbed from those of the standard Gregorian configuration by having different radii of curvature in two principal planes. This improves the off-axis imaging performance by $10 \%$. There is no blockage of the main beam by the secondary reflector or by any support structure.

\section{REFERENCES}

Benford, D.J., Amato, J.J., Mather, J.C., Harvey, S.M.Jr., and Leisawitz, D. 2004, Astrophysics and Space Science 294, 177-212.

Goldsmith, P.F. 1998, Quasioptical Systems: Gaussian Beam Quasioptical Propagation and Applications. (New York: IEEE Press/Chapman Hall), Ch. 6.

Harvey, P.M., Rieke, G.H., Lester, D.F., and Benford, D.J., 2003, Proc. SPIE 4850, 1097-1108.

Lester, D. et al. 2005, Science Promise and Conceptual Mission Design Study for SAFIR - the Single Aperture Far Infrared Observatory. Final Report NASA NRA-03-OSS-01_VM Call for Mission Concepts: Space Science Vision Missions.

Kelsall, T. et al. 1998, Astrophys. J. 508, 44-73 